\title{
Observations on relation-theoretic coincidence theorems under Boyd-Wong type nonlinear contractions
}

\author{
Aftab Alam ${ }^{1 *}$, Mohammad Imdad ${ }^{1}$ and Mohammad Arif ${ }^{1}$
}

*Correspondence:

aafu.amu@gmail.com

'Department of Mathematics,

Aligarh Muslim University, Aligarh, ndia

\begin{abstract}
In this article, we carry out some observations on existing metrical coincidence theorems of Karapinar et al. (Fixed Point Theory Appl. 2014:92, 2014) and Erhan et al. (J. Inequal. Appl. 2015:52, 2015) proved for Lakshmikantham-Ćirić-type nonlinear contractions involving $(f, g)$-closed transitive sets after proving some coincidence theorems satisfying Boyd-Wong-type nonlinear contractivity conditions employing the idea of $(f, g)$-closed locally $f$-transitive binary relation.
\end{abstract}

MSC: $\mathcal{R}$-complete metric spaces; Locally $f$-transitive binary relations; $(g, d)$-self-closedness; $\mathcal{R}$-connectedness

Keywords: $47 \mathrm{H} 10 ; 54 \mathrm{H} 25$

\section{Introduction}

In last ten decades, the classical Banach contraction principle [3] has been generalized by numerous authors in the different directions by improving the underlying contraction conditions (e.g., [4-6]), enhancing the number of involved mappings [4, 7], weakening the involved metrical notions [7, 8], and enlarging the class of ambient spaces [9-11]. In 2004, Ran and Reurings [12] obtained a variant of the classical Banach contraction principle to a complete metric space endowed with partial order relation, which was slightly modified by Nieto and Rodríguez-López [13] in 2005. Later, the trend of utilization of partial order relation in the context of fixed/coincidence point theorems was adopted by various authors $[6,8,14-19]$. Such generalizations are carried out by improving either the contraction conditions or the underlying spaces keeping the partial order relation fixed; however, several authors adopted another way of improving the Banach contraction principle by using various binary relations, such as preorder (Turinici [20]), transitive relation (Ben-El-Mechaiekh [21]), tolerance (Turinici [22, 23]), strict order (Ghods et al. [24]), and symmetric closure (Samet and Turinici [25]).

In 2015, Alam and Imdad [26] obtained yet another generalization of the classical Banach contraction principle employing an amorphous (arbitrary) binary relation and observed that several well-known metrical fixed point theorems can further be improved up to arbitrary binary relations (instead of partial order, preorder, transitive relation, tolerance, strict order, and symmetric closure). With this in mind, Alam and Imdad [7] in-

(c) The Author(s) 2019. This article is distributed under the terms of the Creative Commons Attribution 4.0 International License (http://creativecommons.org/licenses/by/4.0/), which permits unrestricted use, distribution, and reproduction in any medium, provided you give appropriate credit to the original author(s) and the source, provide a link to the Creative Commons license, and indicate if changes were made. 
troduced relation-theoretic analogues of certain involved metrical notions such as completeness, continuity, $g$-continuity, compatibility, etc. and utilized the same to prove coincidence theorems for relation-preserving contractions. It is worth noticing that under the universal relation, such newly defined notions reduce to their corresponding usual notions, and henceforth relation-theoretic metrical fixed/coincidence point theorems reduce to their corresponding classical fixed/coincidence point theorems. Note that relation-preserving contractions remain relatively weaker than usual contractions as they are required to hold merely for the elements that are related in the underlying relation.

Several metrical fixed point theorems under arbitrary binary relations are proved by various authors such as Khan et al. [10], Ayari et al. [27], Roldán-López-de-Hierro [28], Roldán-López-de-Hierro and Shahzad [29], and Shahzad et al. [30], which are generalizations of the relation-theoretic contraction principle due to Alam and Imdad [26]. Here we can point out that arbitrary binary relation is general enough and often does not work for certain contractions, so that various fixed/coincidence point theorems are proved in metric spaces equipped with different types of binary relations, for example, preorder (Roldán-López-de-Hierro and Shahzad [11]), transitive relations (Shahzad et al. [31]), finitely transitive relations (Berzig and Karapinar [32], Berzig et al. [33]), locally finitely transitive relations (Turinici $[34,35]$ ), locally finitely $T$-transitive relations (Alam et al. [36]), and locally $T$-transitive relations (see Alam and Imdad [37]).

In 2010, Samet and Vetro [38] proved coupled fixed point theorems without using partial ordering employing the idea of an $F$-invariant set. In 2013, Kutbi et al. [39] weakened the idea of an $F$-invariant set by introducing the notion of $F$-closed sets. Thereafter Karapinar et al. [1] proved some unidimensional versions of earlier coupled fixed point results involving $F$-closed sets and then obtained such coupled fixed point results by using the corresponding unidimensional fixed point results. Furthermore, Karapinar et al. [1] observed that the notion of a transitive $F$-closed (or $F$-invariant) set is equivalent to the concept of a preordered set and thereafter also showed that some recent multidimensional results using $F$-invariant sets can be reduced to well-known results on ordered metric spaces.

Here we mention two main results of Karapinar et al. [1]. The reader is required to go through Karapinar et al. [1] for relevant notions, such as an $(f, g)$-closed set, an $(f, g)$ compatible set, $M$-continuity, $(O, M)$-compatibility, regularity, a $(g, \preceq)$-increasing mapping, transitive and $g$-transitive sets. In these results, the authors used the following family of control functions utilized by Lakshmikantham and Ćirić [40]:

$$
\Phi=\left\{\varphi:[0, \infty) \rightarrow[0, \infty): \varphi(t)<t \text { for each } t>0 \text { and } \lim _{r \rightarrow t^{+}} \varphi(r)<t \text { for each } t>0\right\} .
$$

Theorem 1 ([1]) Let $(X, d)$ be a complete metric space, let $f, g: X \rightarrow X$ be two mappings, and let $M \subseteq X^{2}$ be a subset such that

(i) $f(X) \subseteq g(X)$,

(ii) $M$ is $(f, g)$-compatible, $(f, g)$-closed, and transitive,

(iii) there exists $x_{0} \in X$ such that $\left(g x_{0}, f x_{0}\right) \in M$,

(iv) there exists $\varphi \in \Phi$ such that

$$
d(f x, f y) \leq \varphi(d(g x, g y)) \quad \forall x, y \in X \text { with }(g x, g y) \in M .
$$


Also assume that at least one of the following conditions holds:

(a) $f$ and $g$ are $M$-continuous and $(O, M)$-compatible,

(b) $f$ and $g$ are continuous and commuting,

(c) $(X, d, M)$ is regular, and $g(X)$ is closed.

Then $f$ and $g$ have at least one coincidence point.

Theorem 2 ([1]) Let $(X, d)$ be a complete metric space, let $\preccurlyeq$ be a transitive relation on $X$, and let $f, g: X \rightarrow X$ be two mappings such that

(i) $f(X) \subseteq g(X)$,

(ii) $f$ is $(g$, $\preccurlyeq)$-increasing,

(iii) there exists $x_{0} \in X$ such that $g\left(x_{0}\right) \preccurlyeq f\left(x_{0}\right)$,

(iv) there exists $\varphi \in \Phi$ such that

$$
d(f x, f y) \leq \varphi(d(g x, g y)) \quad \forall x, y \in X \text { with } g(x) \preccurlyeq g(y),
$$

(v) $\varphi(0)=0$, or $\preccurlyeq$ is antisymmetric.

Assume that either

(a) $f$ and $g$ are continuous and commuting, or

(b) $(X, d, \preccurlyeq)$ is regular, and $g(X)$ is closed.

Then $f$ and $g$ have at least one coincidence point.

Erhan et al. [2] slightly modified Theorem 1 by proving the following sharpened version.

Theorem 3 ([2]) Let $(X, d)$ be a complete metric space, let $f, g: X \rightarrow X$ be two mappings, and let $M \subseteq X^{2}$ be a subset such that

(i) $f(X) \subseteq g(X)$,

(ii) $M$ is $(f, g)$-closed and $g$-transitive,

(iii) there exists $x_{0} \in X$ such that $\left(g x_{0}, f x_{0}\right) \in M$,

(iv) there exists $\varphi \in \Phi$ such that

$$
d(f x, f y) \leq \varphi(d(g x, g y)) \quad \forall x, y \in X \text { with }(g x, g y) \in M .
$$

Also assume that at least one of the following conditions holds:

(a) $f$ and $g$ are $M$-continuous and $(O, M)$-compatible,

(b) $f$ and $g$ are continuous and $(O, M)$-compatible,

(c) $f$ and $g$ are continuous and commuting,

(d) $(X, d, M)$ is regular, $g(X)$ is closed, and $M$ is $(f, g)$-compatible.

Then $f$ and $g$ have at least one coincidence point.

Alam and Imdad [7] observed that relation-theoretic metrical fixed/coincidence point results combine the idea contained in Karapinar et al. [1] as the set $M$ (utilized by Karapinar et al. [1]) being subset of $X^{2}$ is in fact a binary relation on $X$. The aim of this paper is to prove relatively more sharpened and improved versions of foregoing results using a relation-theoretic approach. 


\section{Relation-theoretic notions and auxiliary results}

In this paper, $\mathbb{N}$ and $\mathbb{N}_{0}$ denote the sets of natural numbers and whole numbers, respectively (i.e., $\mathbb{N}_{0}=\mathbb{N} \cup\{0\}$ ). In this section, to make our exposition self-contained, we give some definitions and basic results related to our main results.

Definition 1 ([41]) Let $X$ be a nonempty set. A subset $\mathcal{R}$ of $X^{2}$ is called a binary relation on $X$.

Trivially, $X^{2}$ and $\emptyset$ are binary relations on $X$, which are respectively called the universal relation (or full relation) and empty relation. Another important relation of this kind is the relation $\triangle_{X}=\{(x, x): x \in X\}$, called the identity relation (or the diagonal relation) on $X$.

In this paper, $\mathcal{R}$ stands for a nonempty binary relation, but for simplicity, we write only "binary relation" instead of "nonempty binary relation."

Definition 2 ([26]) Let $\mathcal{R}$ be a binary relation defined on a nonempty set $X$, and let $x, y \in X$. We say that $x$ and $y$ are $\mathcal{R}$-comparative and write $[x, y] \in \mathcal{R}$ if either $(x, y) \in \mathcal{R}$ or $(y, x) \in \mathcal{R}$.

Definition $3([41,42])$ A binary relation $\mathcal{R}$ defined on a nonempty set $X$ is called

- reflexive if $(x, x) \in \mathcal{R} \forall x \in X$,

- irreflexive if $(x, x) \notin \mathcal{R} \forall x \in X$,

- symmetric if $(x, y) \in \mathcal{R}$ implies $(y, x) \in \mathcal{R}$,

- antisymmetric if $(x, y) \in \mathcal{R}$ and $(y, x) \in \mathcal{R}$ imply $x=y$,

- transitive if $(x, y) \in \mathcal{R}$ and $(y, z) \in \mathcal{R}$ imply $(x, z) \in \mathcal{R}$,

- complete or connected or dichotomous if $[x, y] \in \mathcal{R} \forall x, y \in X$,

- weakly complete or weakly connected or trichotomous if $[x, y] \in \mathcal{R}$ or $x=y \forall x, y \in X$.

Definition 4 ([25, 41-45]) A binary relation $\mathcal{R}$ defined on a nonempty set $X$ is called

- amorphous if it has no specific properties at all,

- a strict order or sharp order if $\mathcal{R}$ is irreflexive and transitive,

- a near-order if $\mathcal{R}$ is antisymmetric and transitive,

- a pseudo-order if $\mathcal{R}$ is reflexive and antisymmetric,

- a quasi-order or preorder if $\mathcal{R}$ is reflexive and transitive,

- a partial order if $\mathcal{R}$ is reflexive, antisymmetric, and transitive,

- a simple order if $\mathcal{R}$ is a weakly complete strict order,

- a weak order if $\mathcal{R}$ is a complete preorder,

- a total order or linear order or chain if $\mathcal{R}$ is a complete partial order,

- a tolerance if $\mathcal{R}$ is reflexive and symmetric,

- an equivalence if $\mathcal{R}$ is reflexive, symmetric, and transitive.

Remark 1 Clearly, the universal relation $X^{2}$ on a nonempty set $X$ remains a complete equivalence relation.

Definition 5 ([41]) Let $X$ be a nonempty set, and let $\mathcal{R}$ be a binary relation on $X$.

(1) The inverse or transpose or dual relation of $\mathcal{R}$, denoted by $\mathcal{R}^{-1}$, is defined by $\mathcal{R}^{-1}=\left\{(x, y) \in X^{2}:(y, x) \in \mathcal{R}\right\}$. 
(2) The reflexive closure of $\mathcal{R}$, denoted by $\mathcal{R}^{\#}$, is defined as the set $\mathcal{R} \cup \triangle_{X}$ (i.e., $\mathcal{R}^{\#}:=\mathcal{R} \cup \triangle_{X}$ ). In fact, $\mathcal{R}^{\#}$ is the smallest reflexive relation on $X$ containing $\mathcal{R}$.

(3) The symmetric closure of $\mathcal{R}$, denoted by $\mathcal{R}^{s}$, is defined as the set $\mathcal{R} \cup \mathcal{R}^{-1}$ (i.e., $\left.\mathcal{R}^{s}:=\mathcal{R} \cup \mathcal{R}^{-1}\right)$. In fact, $\mathcal{R}^{s}$ is the smallest symmetric relation on $X$ containing $\mathcal{R}$.

Proposition 1 ([26]) For a binary relation $\mathcal{R}$ defined on a nonempty set $X$,

$$
(x, y) \in \mathcal{R}^{s} \Longleftrightarrow[x, y] \in \mathcal{R} \text {. }
$$

Definition 6 ([46]) Let $X$ be a nonempty set, let $E \subseteq X$, and let $\mathcal{R}$ be a binary relation on $X$. Then the restriction of $\mathcal{R}$ to $E$, denoted by $\left.\mathcal{R}\right|_{E}$, is defined as the set $\mathcal{R} \cap E^{2}$ (i.e., $\left.\mathcal{R}\right|_{E}:=\mathcal{R} \cap E^{2}$ ). In fact, $\left.\mathcal{R}\right|_{E}$ is a relation on $E$ induced by $\mathcal{R}$.

Definition 7 ([26]) Let $X$ be a nonempty set, and let $\mathcal{R}$ be a binary relation on $X$. A sequence $\left\{x_{n}\right\} \subset X$ is called $\mathcal{R}$-preserving if

$$
\left(x_{n}, x_{n+1}\right) \in \mathcal{R} \quad \forall n \in \mathbb{N}_{0} .
$$

Definition 8 ([7]) Let $X$ be a nonempty set, and let $f$ and $g$ be self-mappings on $X$. A binary relation $\mathcal{R}$ defined on $X$ is called $(f, g)$-closed if for all $x, y \in X$,

$$
(g x, g y) \in \mathcal{R} \quad \Rightarrow \quad(f x, f y) \in \mathcal{R}
$$

Note that under the restriction $g=I$, the identity mapping on $X$, Definition 8 reduces to the notion of $f$-closedness of $\mathcal{R}$ defined in [26].

Proposition 2 ([7]) Let $X$ be a nonempty set, let $\mathcal{R}$ be a binary relation on $X$, and let $f$ and $g$ be self-mappings on $X$. If $\mathcal{R}$ is $(f, g)$-closed, then so is $\mathcal{R}^{s}$.

Definition 9 ([7]) Let $(X, d)$ be a metric space, and let $\mathcal{R}$ be a binary relation on $X$. We say that $(X, d)$ is $\mathcal{R}$-complete if every $\mathcal{R}$-preserving Cauchy sequence in $X$ converges.

Remark 2 Every complete metric space is $\mathcal{R}$-complete for any binary relation $\mathcal{R}$. Particularly, under the universal relation, the notion of $\mathcal{R}$-completeness coincides with usual completeness.

Definition 10 ([7]) Let $(X, d)$ be a metric space, and let $\mathcal{R}$ be binary relation on $X$. A subset $E$ of $X$ is called $\mathcal{R}$-closed if every $\mathcal{R}$-preserving convergent sequence in $E$ converges to a point of $E$.

Remark 3 Every closed subset of a metric space is $\mathcal{R}$-closed for any binary relation $\mathcal{R}$. Particularly, under the universal relation, the notion of $\mathcal{R}$-closedness coincides with usual closedness.

Proposition 3 ([7]) An $\mathcal{R}$-complete subspace of a metric space is $\mathcal{R}$-closed.

Proposition 4 ([7]) An $\mathcal{R}$-closed subspace of an $\mathcal{R}$-complete metric space is $\mathcal{R}$-complete. 
Definition 11 ([7]) Let $(X, d)$ be a metric space, let $\mathcal{R}$ be a binary relation on $X$, let $g$ be a self-mapping on $X$, and let $x \in X$. A mapping $f: X \rightarrow X$ is called $(g, \mathcal{R})$-continuous at $x$ if for any sequence $\left\{x_{n}\right\}$ such that $\left\{g x_{n}\right\}$ is $\mathcal{R}$-preserving and $g\left(x_{n}\right) \stackrel{d}{\longrightarrow} g(x)$, we have $f\left(x_{n}\right) \stackrel{d}{\longrightarrow} f(x)$. Moreover, $f$ is called $(g, \mathcal{R})$-continuous if it is $(g, \mathcal{R})$-continuous at each point of $X$.

Note that under the restriction $g=I$, the identity mapping on $X$, Definition 11 reduces to the notion of $\mathcal{R}$-continuity of $f$ defined in [7].

Remark 4 Every continuous (respectively, $g$-continuous) mapping is $\mathcal{R}$-continuous (respectively, $(g, \mathcal{R})$-continuous) for any binary relation $\mathcal{R}$. Particularly, under the universal relation, the notion of $\mathcal{R}$-continuity (respectively, $(g, \mathcal{R})$-continuity) coincides with usual continuity (respectively, $g$-continuity).

Definition 12 ([7]) Let $(X, d)$ be a metric space, let $\mathcal{R}$ be a binary relation on $X$, and let $f$ and $g$ be self-mappings on $X$. We say that the mappings $f$ and $g$ are $\mathcal{R}$-compatible if for any sequence $\left\{x_{n}\right\} \subset X$ such that $\left\{x_{n}\right\}$ and $\left\{g x_{n}\right\}$ are $\mathcal{R}$-preserving and $\lim _{n \rightarrow \infty} g\left(x_{n}\right)=$ $\lim _{n \rightarrow \infty} f\left(x_{n}\right)$, we have

$$
\lim _{n \rightarrow \infty} d\left(g f x_{n}, f g x_{n}\right)=0
$$

Remark 5 In a metric space $(X, d)$ endowed with a binary relation $\mathcal{R}$,

$$
\text { commutativity } \Rightarrow \text { compatibility } \Rightarrow \mathcal{R} \text {-compatibility. }
$$

Particularly, under the universal relation, the notion of $\mathcal{R}$-compatibility coincides with usual compatibility.

Definition 13 ([7]) Let $(X, d)$ be a metric space, and let $g$ be a self-mapping on $X$. A binary relation $\mathcal{R}$ defined on $X$ is called $(g, d)$-self-closed if for any $\mathcal{R}$-preserving sequence $\left\{x_{n}\right\}$ such that $x_{n} \stackrel{d}{\longrightarrow} x$, there exists a subsequence $\left\{x_{n_{k}}\right\}$ of $\left\{x_{n}\right\}$ with $\left[g x_{n_{k}}, g x\right] \in \mathcal{R}$ for all $k \in \mathbb{N}_{0}$.

Note that under the restriction $g=I$, the identity mapping on $X$, Definition 13 reduces to the notion of $d$-self-closedness of $\mathcal{R}$ defined in [26].

Definition 14 ([47]) Given a mapping $f: X \rightarrow X$, a binary relation $\mathcal{R}$ defined on $X$ is called $f$-transitive if for any $x, y, z \in X$,

$$
(f x, f y),(f y, f z) \in \mathcal{R} \quad \Rightarrow \quad(f x, f z) \in \mathcal{R} .
$$

Inspired by Turinici [34, 35], Alam and Imdad [37] introduced the following notion by localizing the notion of $f$-transitivity.

Definition 15 ([37]) Let $X$ be a nonempty set, and let $f$ be a self-mapping on $X$. A binary relation $\mathcal{R}$ on $X$ is called locally $f$-transitive if for each (effectively) $\mathcal{R}$-preserving sequence $\left\{x_{n}\right\} \subset f(X)$ (with range $E:=\left\{x_{n}: n \in \mathbb{N}_{0}\right\}$ ), the binary relation $\left.\mathcal{R}\right|_{E}$ is transitive. 
Clearly, for a given a self-mapping $f$ and a binary relation $\mathcal{R}$ on a nonempty set $X$,

$$
\text { transitivity } \Rightarrow f \text {-transitivity } \Rightarrow \text { locally } f \text {-transitivity. }
$$

Definition 16 ([25]) Let $X$ be a nonempty set, and let $\mathcal{R}$ be a binary relation on $X$. A subset $E$ of $X$ is called $\mathcal{R}$-directed if for each pair $x, y \in E$, there exists $z \in X$ such that $(x, z) \in \mathcal{R}$ and $(y, z) \in \mathcal{R}$.

Definition 17 ([46]) Let $X$ be a nonempty set, and let $\mathcal{R}$ be a binary relation on $X$. For $x, y \in X$, a path of length $k$ (where $k$ is a natural number) in $\mathcal{R}$ from $x$ to $y$ is a finite sequence $\left\{z_{0}, z_{1}, z_{2}, \ldots, z_{k}\right\} \subset X$ satisfying the following conditions:

(i) $z_{0}=x$ and $z_{k}=y$,

(ii) $\left(z_{i}, z_{i+1}\right) \in \mathcal{R}$ for each $i(0 \leq i \leq k-1)$.

Note that a path of length $k$ involves $k+1$ elements of $X$ although they are not necessarily distinct.

Definition 18 ([7]) Let $X$ be a nonempty set, and let $\mathcal{R}$ be a binary relation on $X$. A subset $E$ of $X$ is called $\mathcal{R}$-connected if for each pair $x, y \in E$, there exists a path in $\mathcal{R}$ from $x$ to $y$.

Inspired by the notion of an $(f, g)$-compatible subset of $X^{2}$ utilized by Karapinar et al. [1] and Roldán-López-de-Hierro et al. [47], we introduce the following notion.

Definition 19 Let $X$ be a nonempty set, and let $f$ and $g$ be self-mappings on $X$. A binary relation $\mathcal{R}$ defined on $X$ is called $(f, g)$-compatible if for all $x, y \in X$,

$$
(g x, g y) \in \mathcal{R} \quad \text { and } \quad g(x)=g(y) \quad \Rightarrow \quad f(x)=f(y) .
$$

Next, we propose the following fact.

Proposition 5 Let $X$ be a nonempty set, let $\mathcal{R}$ be a binary relation on $X$, and let $f$ and $g$ be self-mappings on $X$.If $\mathcal{R}$ is antisymmetric and $(f, g)$-closed, then $\mathcal{R}$ is $(f, g)$-compatible.

Proof Take $x, y \in X$ such that $(g x, g y) \in \mathcal{R}$ and $g(x)=g(y)$, which yields that

$$
(g x, g y) \in \mathcal{R} \quad \text { and } \quad(g y, g x) \in \mathcal{R} .
$$

As $\mathcal{R}$ is $(f, g)$-closed, we have

$$
(f x, f y) \in \mathcal{R} \quad \text { and } \quad(f y, f x) \in \mathcal{R},
$$

which by the antisymmetry of $\mathcal{R}$ implies that

$$
f(x)=f(y) .
$$

For a given binary relation $\mathcal{R}$ and two self-mappings $f$ and $g$ defined on a nonempty set $X, X(f, g, \mathcal{R})$ denotes the subset $\{x \in X:(g x, f x) \in \mathcal{R}\}$ of $X$.

Recently, Alam and Imdad [7] proved the following relation-theoretic coincidence theorem under linear contraction. 
Theorem 4 ([7]) Let $(X, d)$ be a metric space, let $\mathcal{R}$ be a binary relation on $X$, and let $f$ and $g$ be self-mappings on $X$. Let $Y$ be an $\mathcal{R}$-complete subspace of $X$. Suppose that the following conditions hold:

(a) $f(X) \subseteq g(X) \cap Y$,

(b) $\mathcal{R}$ is $(f, g)$-closed,

(c) $X(f, g, \mathcal{R})$ is nonempty,

(d) there exists $\alpha \in[0,1)$ such that

$$
d(f x, f y) \leq \alpha d(g x, g y) \quad \forall x, y \in X \text { with }(g x, g y) \in \mathcal{R},
$$

(e) (e1) $f$ and $g$ are $\mathcal{R}$-compatible,

(e2) $g$ is $\mathcal{R}$-continuous,

(e3) either $f$ is $\mathcal{R}$-continuous, or $\mathcal{R}$ is $(g, d)$-self-closed,

or, alternatively,

(e') $\quad\left(e^{\prime} 1\right) \quad Y \subseteq g(X)$

$\left(e^{\prime} 2\right)$ either $f$ is $(g, \mathcal{R})$-continuous, or $f$ and $g$ are continuous, or $\left.\mathcal{R}\right|_{Y}$ is $d$-self-closed.

Then $f$ and $g$ have a coincidence point.

The following family of control functions is indicated by Boyd and Wong [48] but was later used by Jotic [49]:

$$
\Omega=\left\{\varphi:[0, \infty) \rightarrow[0, \infty): \varphi(t)<t \text { for each } t>0 \text { and } \limsup _{r \rightarrow t^{+}} \varphi(r)<t \text { for each } t>0\right\} .
$$

Clearly, the family $\Omega$ enlarges the family $\Phi$, i.e., $\Phi \subset \Omega$. By the symmetry of $d$ we have the following:

Proposition 6 If $(X, d)$ is a metric space, $\mathcal{R}$ is a binary relation on $X, f$ and $g$ are selfmappings on $X$, and $\varphi \in \Omega$, then the following contractivity conditions are equivalent:

(I) $\quad d(f x, f y) \leq \varphi(d(g x, g y)) \quad \forall x, y \in X$ with $(g x, g y) \in \mathcal{R}$,

(II) $\quad d(f x, f y) \leq \varphi(d(g x, g y)) \quad \forall x, y \in X$ with $[g x, g y] \in \mathcal{R}$.

Finally, we state the following known results, which are needed in the proofs of our main results.

Lemma 1 ([18]) Let $\varphi \in \Omega$. If $\left\{a_{n}\right\} \subset(0, \infty)$ is a sequence such that $a_{n+1} \leq \varphi\left(a_{n}\right)$ for all $n \in \mathbb{N}_{0}$, then $\lim _{n \rightarrow \infty} a_{n}=0$.

Lemma $2([33,35])$ Let $(X, d)$ be a metric space, and let $\left\{x_{n}\right\}$ be a sequence in $X$. If $\left\{x_{n}\right\}$ is not a Cauchy sequence, then there exist $\epsilon>0$ and two subsequences $\left\{x_{n_{k}}\right\}$ and $\left\{x_{m_{k}}\right\}$ of $\left\{x_{n}\right\}$ such that

(i) $k \leq m_{k}<n_{k} \forall k \in \mathbb{N}$,

(ii) $d\left(x_{m_{k}}, x_{n_{k}}\right)>\epsilon \forall k \in \mathbb{N}$,

(iii) $d\left(x_{m_{k}}, x_{n_{k-1}}\right) \leq \epsilon \forall k \in \mathbb{N}$.

Moreover, suppose that $\lim _{n \rightarrow \infty} d\left(x_{n}, x_{n+1}\right)=0$. Then

(iv) $\lim _{k \rightarrow \infty} d\left(x_{m_{k}}, x_{n_{k}}\right)=\epsilon$,

(v) $\lim _{k \rightarrow \infty} d\left(x_{m_{k}+1}, x_{n_{k}+1}\right)=\epsilon$. 
Lemma 3 ([50]) Let $X$ be a nonempty set, and let $g$ be a self-mapping on $X$. Then there exists a subset $E \subseteq X$ such that $g(E)=g(X)$ and $g: E \rightarrow X$ is one-to-one.

\section{Main results}

Now, we are equipped to prove the following result on the existence of a coincidence point under the $\varphi$-contractivity condition.

Theorem 5 Let $(X, d)$ be a metric space, let $\mathcal{R}$ be a binary relation on $X$, and let $Y$ be an $\mathcal{R}$-complete subspace of $X$. Let $f$ and $g$ be self-mappings on $X$. Suppose that the following conditions hold:

(a) $f(X) \subseteq g(X) \cap Y$,

(b) $\mathcal{R}$ is $(f, g)$-closed and locally $f$-transitive,

(c) $X(f, g, \mathcal{R})$ is nonempty,

(d) there exists $\varphi \in \Omega$ such that

$$
d(f x, f y) \leq \varphi(d(g x, g y)) \quad \forall x, y \in X \text { with }(g x, g y) \in \mathcal{R},
$$

(e) (e1) $f$ and $g$ are $\mathcal{R}$-compatible,

(e2) $g$ is $\mathcal{R}$-continuous,

(e3) either $f$ is $\mathcal{R}$-continuous, or $\mathcal{R}$ is $(f, g)$-compatible and $(g, d)$-self-closed, or, alternatively,

$\left(e^{\prime}\right) \quad\left(e^{\prime} 1\right) \quad Y \subseteq g(X)$,

$\left(e^{\prime} 2\right)$ either $f$ is $(g, \mathcal{R})$-continuous, or $f$ and $g$ are continuous, or $\mathcal{R}$ and $\left.\mathcal{R}\right|_{Y}$ are $(f, g)$-compatible and $d$-self-closed, respectively.

Then $f$ and $g$ have a coincidence point.

Proof Firstly, we notice that assumption (a) is equivalent to $f(X) \subseteq g(X)$ and $f(X) \subseteq Y$. Now, in view of assumption $(c)$, let $x_{0}$ be an arbitrary element of $X(f, g, \mathcal{R})$ such that $\left(g x_{0}, f x_{0}\right) \in \mathcal{R}$. If $g\left(x_{0}\right)=f\left(x_{0}\right)$, then $x_{0}$ is a coincidence point of $f$ and $g$, and hence the proof is finished. Otherwise, using the assumption $f(X) \subseteq g(X)$, we construct a sequence $\left\{x_{n}\right\} \subset X$ of joint iteration of $f$ and $g$ based at point $x_{0}$, that is,

$$
g\left(x_{n+1}\right)=f\left(x_{n}\right) \quad \forall n \in \mathbb{N}_{0} .
$$

Now we assert that $\left\{g x_{n}\right\}$ is an $\mathcal{R}$-preserving sequence, that is,

$$
\left(g x_{n}, g x_{n+1}\right) \in \mathcal{R} \quad \forall n \in \mathbb{N}_{0} .
$$

We prove this fact by mathematical induction. Using Eq. (1) (with $n=0)$ and the fact that $x_{0} \in X(f, g, \mathcal{R})$, we have

$$
\left(g x_{0}, g x_{1}\right) \in \mathcal{R}
$$

Thus (2) holds for $n=0$. Assume that (2) holds for $n=r>0$, that is,

$$
\left(g x_{r}, g x_{r+1}\right) \in \mathcal{R} \text {. }
$$


Since $\mathcal{R}$ is $(f, g)$-closed, we have

$$
\left(f x_{r}, f x_{r+1}\right) \in \mathcal{R}
$$

which by (1) implies

$$
\left(g x_{r+1}, g x_{r+2}\right) \in \mathcal{R}
$$

that is, (2) holds for $n=r+1$. Hence, by induction, (2) holds for all $n \in \mathbb{N}_{0}$.

By (1) and (2) the sequence $\left\{f x_{n}\right\}$ is also $\mathcal{R}$-preserving, that is,

$$
\left(f x_{n}, f x_{n+1}\right) \in \mathcal{R} \quad \forall n \in \mathbb{N}_{0} .
$$

If $g\left(x_{n_{0}}\right)=g\left(x_{n_{0}+1}\right)$ for some $n_{0} \in \mathbb{N}$, then using (1), we have $g\left(x_{n_{0}}\right)=f\left(x_{n_{0}}\right)$, that is, $x_{n_{0}}$ is a coincidence point of $f$ and $g$, so that we are done. On the other hand, if $g\left(x_{n}\right) \neq g\left(x_{n+1}\right)$ for each $n \in \mathbb{N}_{0}$, then we can define a sequence $\left\{d_{n}\right\}_{n=0}^{\infty} \subset(0, \infty)$ by

$$
d_{n}:=d\left(g x_{n}, g x_{n+1}\right) .
$$

Applying (1), (2), (4), and assumption (d), we deduce that

$$
d_{n+1}=d\left(g x_{n+1}, g x_{n+2}\right)=d\left(f x_{n}, f x_{n+1}\right) \leq \varphi\left(d\left(g x_{n}, g x_{n+1}\right)\right)=\varphi\left(d_{n}\right),
$$

so that

$$
d_{n+1} \leq \varphi\left(d_{n}\right)
$$

which by Lemma 1 implies

$$
\lim _{n \rightarrow \infty} d_{n}=\lim _{n \rightarrow \infty} d\left(g x_{n}, g x_{n+1}\right)=0 .
$$

Now we show that $\left\{g x_{n}\right\}$ is a Cauchy sequence. On the contrary, assume that $\left\{g x_{n}\right\}$ is not Cauchy. Therefore by Lemma 2 there exist $\epsilon>0$ and two subsequences $\left\{g x_{n_{k}}\right\}$ and $\left\{g x_{m_{k}}\right\}$ of $\left\{g x_{n}\right\}$ such that

$$
k \leq m_{k}<n_{k}, \quad d\left(g x_{m_{k}}, g x_{n_{k}}\right)>\epsilon \geq d\left(g x_{m_{k}}, g x_{n_{k-1}}\right) \quad \forall k \in \mathbb{N} .
$$

Further, in view of (5) and Lemma 2, we have

$$
\lim _{n \rightarrow \infty} d\left(g x_{m_{k}}, g x_{n_{k}}\right)=\lim _{n \rightarrow \infty} d\left(g x_{m_{k+1}}, g x_{n_{k+1}}\right)=\epsilon .
$$

Denote $\eta_{k}:=d\left(g x_{m_{k}}, g x_{n_{k}}\right)$. As $\left\{g x_{n}\right\}$ is $\mathcal{R}$-preserving (owing to (2)) and $\left\{g x_{n}\right\} \subseteq f(X)$ (owing to (1)), by the local $f$-transitivity of $\mathcal{R}$ we have $\left(g x_{m_{k}}, g x_{n_{k}}\right) \in \mathcal{R}$. Hence, applying contractivity condition $(d)$, we obtain

$$
d\left(g x_{m_{k+1}}, g x_{n_{k+1}}\right)=d\left(f x_{m_{k}}, f x_{n_{k}}\right) \leq \varphi\left(d\left(g x_{m_{k}}, g x_{n_{k}}\right)\right)=\varphi\left(\eta_{k}\right),
$$


so that

$$
d\left(g x_{m_{k+1}}, g x_{n_{k+1}}\right) \leq \varphi\left(\eta_{k}\right) .
$$

Since $\eta_{k} \rightarrow \epsilon$ in the real line as $k \rightarrow \infty$ (owing to (7)) and $\eta_{k}>\epsilon$ for all $k \in \mathbb{N}$ (owing to (6)), by the definition of $\Omega$, we have

$$
\limsup _{k \rightarrow \infty} \varphi\left(\eta_{k}\right)=\limsup _{r \rightarrow \epsilon^{+}} \varphi(r)<\epsilon .
$$

Taking the limit as $k \rightarrow \infty$ in (8) and using (7) and (9), we obtain

$$
\epsilon=\limsup _{k \rightarrow \infty} d\left(g x_{m_{k+1}}, g x_{n_{k+1}}\right) \leq \limsup _{k \rightarrow \infty} \varphi\left(\eta_{k}\right)<\epsilon,
$$

which is a contradiction, so that $\left\{g x_{n}\right\}$ is a Cauchy sequence. By (1), $\left\{g x_{n}\right\} \subset f(X) \subseteq Y$, so that $\left\{g x_{n}\right\}$ is an $\mathcal{R}$-preserving Cauchy sequence in $Y$. As $Y$ is $\mathcal{R}$-complete, there exists $z \in Y$ such that $\lim _{n \rightarrow \infty} g\left(x_{n}\right)=z$, which, together with (2), implies

$$
\lim _{n \rightarrow \infty} g\left(x_{n}\right)=z .
$$

Applying (1) and (10), we obtain

$$
\lim _{n \rightarrow \infty} f\left(x_{n}\right)=z .
$$

Now we complete the proof by using $(e)$ and $\left(e^{\prime}\right)$. Assume that (e) holds. Using (2), (10), and assumption $(e 2)$ (i.e., the $\mathcal{R}$-continuity of $g$ ), we have

$$
\lim _{n \rightarrow \infty} g\left(g x_{n}\right)=g\left(\lim _{n \rightarrow \infty} g x_{n}\right)=g(z) .
$$

Now, utilizing (3), (11), and assumption (e2) (i.e., the $\mathcal{R}$-continuity of $g$ ), we have

$$
\lim _{n \rightarrow \infty} g\left(f x_{n}\right)=g\left(\lim _{n \rightarrow \infty} f x_{n}\right)=g(z) .
$$

Since $\left\{x_{n}\right\}$ and $\left\{g x_{n}\right\}$ are $\mathcal{R}$-preserving (owing to (2) and (3)) and $\lim _{n \rightarrow \infty} f\left(x_{n}\right)=$ $\lim _{n \rightarrow \infty} g\left(x_{n}\right)=z$ (owing to (10) and (11)), assumption (e1) (i.e., the $\mathcal{R}$-compatibility of $f$ and $g$ ), implies

$$
\lim _{n \rightarrow \infty} d\left(g f x_{n}, f g x_{n}\right)=0 .
$$

Now we prove that $z$ is a coincidence point of $f$ and $g$. To substantiate this, we use assumption (e3). Assume that $f$ is $\mathcal{R}$-continuous. Using (2), (10), and the $\mathcal{R}$-continuity of $f$, we obtain

$$
\lim _{n \rightarrow \infty} f\left(g x_{n}\right)=f\left(\lim _{n \rightarrow \infty} g x_{n}\right)=f(z) .
$$

Applying (13), (14), (15), and the continuity of $d$, we obtain

$$
d(g z, f z)=d\left(\lim _{n \rightarrow \infty} g f x_{n}, \lim _{n \rightarrow \infty} f g x_{n}\right)=\lim _{n \rightarrow \infty} d\left(g f x_{n}, f g x_{n}\right)=0,
$$


so that

$$
g(z)=f(z) .
$$

Thus $z$ is a coincidence point of $f$ and $g$, and hence we are through. Alternately, assume that $\mathcal{R}$ is $(g, d)$-self-closed and $(f, g)$-compatible. As $\left\{g x_{n}\right\}$ is $\mathcal{R}$-preserving (due to (2)) and $g\left(x_{n}\right) \stackrel{d}{\longrightarrow} z$ (in view of (10)), due to the $(g, d)$-self-closedness of $\mathcal{R}$, there exists a subsequence $\left\{g x_{n_{k}}\right\}$ of $\left\{g x_{n}\right\}$ such that

$$
\left[g g x_{n_{k}}, g z\right] \in \mathcal{R} \quad \forall k \in \mathbb{N}_{0}
$$

Since $g\left(x_{n_{k}}\right) \stackrel{d}{\longrightarrow} z$, Eqs. (10)-(14) also hold for $\left\{x_{n_{k}}\right\}$ (instead of $\left.\left\{x_{n}\right\}\right)$. Using (16), assumption $(d)$, and Proposition 6, we obtain

$$
d\left(f g x_{n_{k}}, f z\right) \leq \varphi\left(d\left(g g x_{n_{k}}, g z\right)\right) \quad \forall k \in \mathbb{N}_{0} .
$$

Now we claim that

$$
d\left(f g x_{n_{k}}, f z\right) \leq d\left(g g x_{n_{k}}, g z\right) \quad \forall k \in \mathbb{N} .
$$

Since there arise two different possibilities, we consider a partition $\left\{\mathbb{N}^{0}, \mathbb{N}^{+}\right\}$of $\mathbb{N}$ (i.e., $\mathbb{N}^{0} \cup$ $\mathbb{N}^{+}=\mathbb{N}$ and $\mathbb{N}^{0} \cap \mathbb{N}^{+}=\emptyset$ ) such that

(i) $d\left(g g x_{n_{k}}, g z\right)=0 \forall k \in \mathbb{N}^{0}$, and

(ii) $d\left(g g x_{n_{k}}, g z\right)>0 \forall k \in \mathbb{N}^{+}$.

In case (i), using (16) and the $(f, g)$-compatibility of $\mathcal{R}$, we get $d\left(f g x_{n_{k}}, f z\right)=0$ for all $k \in \mathbb{N}^{0}$, so that (17) holds for all $k \in \mathbb{N}^{0}$. In case (ii), by the definition of $\Omega$ we have $d\left(f g x_{n_{k}}, f z\right) \leq$ $\varphi\left(d\left(g g x_{n_{k}}, g z\right)\right)<d\left(g g x_{n_{k}}, g z\right)$ for all $k \in \mathbb{N}^{+}$, and hence (17) holds for all $k \in \mathbb{N}^{+}$, so that (17) holds for all $k \in \mathbb{N}$. Using the triangle inequality, (12), (13), (14), and (17), we get

$$
\begin{aligned}
d(g z, f z) & \leq d\left(g z, g f x_{n_{k}}\right)+d\left(g f x_{n_{k}}, f g x_{n_{k}}\right)+d\left(f g x_{n_{k}}, f z\right) \\
& \leq d\left(g z, g f x_{n_{k}}\right)+d\left(g f x_{n_{k}}, f g x_{n_{k}}\right)+d\left(g g x_{n_{k}}, g z\right) \\
& \rightarrow 0 \quad \text { as } k \rightarrow \infty,
\end{aligned}
$$

so that

$$
g(z)=f(z) .
$$

Thus $z$ is a coincidence point of $f$ and $g$, and hence we are done.

Now, assume that $\left(e^{\prime}\right)$ holds. By assumption $\left(e^{\prime} 1\right)$ (i.e., $\left.Y \subseteq g(X)\right)$ we can find $u \in X$ such that $z=g(u)$. Hence (10) and (11), respectively, reduce to

$$
\begin{aligned}
& \lim _{n \rightarrow \infty} g\left(x_{n}\right)=g(u), \\
& \lim _{n \rightarrow \infty} f\left(x_{n}\right)=g(u) .
\end{aligned}
$$


Now we prove that $u$ is a coincidence point of $f$ and $g$. To do this, we use assumption $\left(e^{\prime} 2\right)$. Firstly, assume that $f$ is $(g, \mathcal{R})$-continuous. Then using (18), we get

$$
\lim _{n \rightarrow \infty} f\left(x_{n}\right)=f(u) .
$$

Using (19) and (20), we get

$$
g(u)=f(u)
$$

and hence we are done. Secondly, assume that $f$ and $g$ are continuous. By Lemma 3 there exists a subset $E \subseteq X$ such that $g(E)=g(X)$ and $g: E \rightarrow X$ is one-to-one. Now define $T$ : $g(E) \rightarrow g(X)$ by

$$
T(g a)=f(a) \quad \forall g(a) \in g(E) \text { where } a \in E .
$$

Since $g: E \rightarrow X$ is one-to-one and $f(X) \subseteq g(X), T$ is well defined. Again, since $f$ and $g$ are continuous, it follows that $T$ is continuous. Using $g(X)=g(E)$, assumptions $(a)$ and $\left(e^{\prime} 1\right)$, respectively, reduce to $f(X) \subseteq g(E) \cap Y$ and $Y \subseteq g(E)$, which implies that, without loss of generality, we are able to construct $\left\{x_{n}\right\}_{n=1}^{\infty} \subset E$ satisfying (1) and enabling us to choose $u \in E$. Using (18), (19), (21), and the continuity of $T$, we get

$$
f(u)=T(g u)=T\left(\lim _{n \rightarrow \infty} g x_{n}\right)=\lim _{n \rightarrow \infty} T\left(g x_{n}\right)=\lim _{n \rightarrow \infty} f\left(x_{n}\right)=g(u) .
$$

Thus $u$ is a coincidence point of $f$ and $g$, and hence we are through. Finally, assume that $\mathcal{R}$ and $\left.\mathcal{R}\right|_{Y}$ are $(f, g)$-compatible and $d$-self-closed, respectively. Since $\left\{g x_{n}\right\}$ is $\left.\mathcal{R}\right|_{Y^{-}}$ preserving (due to (2)) and $g\left(x_{n}\right) \stackrel{d}{\longrightarrow} g(u) \in Y$ (due to (18)), by the $d$-self-closeness of $\left.\mathcal{R}\right|_{Y}$ there exists a subsequence $\left\{g x_{n_{k}}\right\}$ of $\left\{g x_{n}\right\}$ such that

$$
\left.\left[g x_{n_{k}}, g u\right] \in \mathcal{R}\right|_{Y} \quad \forall k \in \mathbb{N}_{0}
$$

Applying (1), (22), assumption (d), and Proposition 6, we obtain

$$
d\left(g x_{n_{k}+1}, f u\right)=d\left(f x_{n_{k}}, f u\right) \leq \varphi\left(d\left(g x_{n_{k}}, g u\right)\right) \quad \forall k \in \mathbb{N}_{0} .
$$

We claim that

$$
d\left(g x_{n_{k}+1}, f u\right) \leq d\left(g x_{n_{k}}, g u\right) \quad \forall k \in \mathbb{N} .
$$

Since there arise two different possibilities, we consider a partition $\left\{\mathbb{N}^{0}, \mathbb{N}^{+}\right\}$of $\mathbb{N}$ (i.e., $\mathbb{N}^{0} \cup$ $\mathbb{N}^{+}=\mathbb{N}$ and $\mathbb{N}^{0} \cap \mathbb{N}^{+}=\emptyset$ ) such that

(i) $d\left(g x_{n_{k}}, g u\right)=0 \forall k \in \mathbb{N}^{0}$, and

(ii) $d\left(g x_{n_{k}}, g u\right)>0 \forall k \in \mathbb{N}^{+}$.

In case (i), using (22) and the $(f, g)$-compatibility of $\mathcal{R}$, we get $d\left(f x_{n_{k}}, f u\right)=0$ for all $k \in \mathbb{N}^{0}$, which by (1) implies $d\left(g x_{n_{k}+1}, f u\right)=0$ for all $k \in \mathbb{N}^{0}$, and hence (23) holds for all $k \in \mathbb{N}^{0}$. In case (ii), by the definition of $\Omega$ we have $d\left(g x_{n_{k}+1}, f u\right) \leq \varphi\left(d\left(g x_{n_{k}}, g u\right)\right)<d\left(g x_{n_{k}}, g u\right)$ for all $k \in \mathbb{N}^{+}$, and hence (23) holds for all $k \in \mathbb{N}^{+}$. Thus (23) holds for all $k \in \mathbb{N}$. 
Applying (18), (23), and the continuity of $d$, we get

$$
\begin{aligned}
d(g u, f u) & =d\left(\lim _{k \rightarrow \infty} g x_{n_{k}+1}, f u\right) \\
& =\lim _{k \rightarrow \infty} d\left(g x_{n_{k}+1}, f u\right) \\
& \leq \lim _{k \rightarrow \infty} d\left(g x_{n_{k}}, g u\right) \\
& =0,
\end{aligned}
$$

so that

$$
g(u)=f(u) .
$$

Hence $u$ is a coincidence point of $f$ and $g$, which completes the proof.

Corollary 1 Let $X$ be a nonempty set endowed with a binary relation $\mathcal{R}$ and a metric $d$ such that the metric space $(X, d)$ is $\mathcal{R}$-complete. Let and $f$ and $g$ be self-mappings on $X$. Assume that the following conditions hold:

(a) $f(X) \subseteq g(X)$,

(b) $\mathcal{R}$ is $(f, g)$-closed and locally $f$-transitive,

(c) $X(f, g, \mathcal{R})$ is nonempty,

(d) there exists $\varphi \in \Omega$ such that

$$
d(f x, f y) \leq \varphi(d(g x, g y)) \quad \forall x, y \in X \text { with }(g x, g y) \in \mathcal{R},
$$

(e) (e1) $f$ and $g$ are $\mathcal{R}$-compatible,

(e2) $g$ is $\mathcal{R}$-continuous,

(e3) either $f$ is $\mathcal{R}$-continuous, or $\mathcal{R}$ is $(g, d)$-self-closed and $(f, g)$-compatible, or, alternatively,

(e') (e'1) there exists an $\mathcal{R}$-closed subspace $Y$ of $X$ such that $f(X) \subseteq Y \subseteq g(X)$,

$\left(e^{\prime} 2\right)$ either $f$ is $(g, \mathcal{R})$-continuous, or $f$ and $g$ are continuous, or $\mathcal{R}$ and $\left.\mathcal{R}\right|_{Y}$ are $(f, g)$-compatible and $d$-self-closed, respectively.

Then $f$ and $g$ have a coincidence point.

Proof The result corresponding to part (e) and alternating part $\left(e^{\prime}\right)$ follows by taking $Y=X$ in Theorem 5 and using Proposition 4, respectively.

Remark 6 If $g$ is onto in Corollary 1 , then we can remove assumption $(a)$ as in this case it trivially holds. Also, we can omit assumption $\left(e^{\prime} 1\right)$ as it trivially hods for $Y=g(X)=X$ using Proposition 3. Whenever $f$ is onto, in view of assumption (a), $g$ must be onto, and hence again the same conclusion is immediate.

Remark 7 Firstly, we notice that Theorem 3 is an improved version of Theorem 1, Also, in Theorem 3, assumptions $(b)$ and (c) directly follow from $(a)$. With $\mathcal{R}=M$, Corollary 1 remains a sharpened version of Theorem 3 , which is worth noting with the following respects:

- The notion of "locally $f$-transitive binary relation" is weaker than that of "transitive/g-transitive binary relation." 
- The notion of "regularity of $(X, d, M)$ " (in the context of hypothesis $(d)$ of Theorem 3) can be replaced by a relatively weaker notion, namely " $(g, d)$-self-closedness of $M$." Further, the notion of " $(g, d)$-self-closedness of $M$ " is not necessary as it can also alternatively be replaced by either " $(g, M)$-continuity of $f$ " or "continuity of $f$ and $g$."

- The notion of " $(O, M)$-compatibility of $f$ and $g$ " is replaced by a relatively weaker notion, namely " $M$-compatibility of $f$ and $g$."

- There is no need to impose the closedness requirement on $g(X)$ as it suffices to take an $M$-closed subspace $Y$ of $X$ such that $f(X) \subseteq Y \subseteq g(X)$.

Corollary 2 Theorem 5 (also Corollary 1 ) remains true if we replace the assumption " $(f, g)$ compatibility of $\mathcal{R}$ " (utilized in assumptions $(e 3)$ and $\left(e^{\prime} 2\right)$ ) by one of the following conditions while retaining the rest of the hypotheses:

(i) $\varphi(0)=0$,

(ii) $g$ is one-to-one,

(iii) $\mathcal{R}$ is antisymmetric.

Proof Suppose that (i) holds. Take $x, y \in X$ such that $(g x, g y) \in \mathcal{R}$ and $g(x)=g(y)$. Utilizing the contractivity condition $(d)$, we get $d(f x, f y) \leq \varphi(0)=0$, which implies that $f(x)=f(y)$. It follows that $\mathcal{R}$ is $(f, g)$-compatible.

Suppose that (ii) holds. Take $x, y \in X$ such that $(g x, g y) \in \mathcal{R}$ and $g(x)=g(y)$. As $g$ is oneto-one, we get $x=y$, which implies that $f(x)=f(y)$. It follows that $\mathcal{R}$ is $(f, g)$-compatible.

Finally, in case (iii), our result follows from Proposition 5.

Remark 8 Corollary 2 with $\mathcal{R}:=\preccurlyeq$ remains an improved version of Theorem 2 in the following respects:

- " $(f, g)$-closedness of $\preccurlyeq$ ” is equivalent to " $(g, \preccurlyeq)$-increasingness of $f$ ";

- "locally $f$-transitivity of $\preccurlyeq$ " is weaker than "transitivity of $\preccurlyeq$ ";

- hypothesis (v) (of Theorem 2) can also be replaced by the injectivity of $g$;

- "commutativity of $f$ and $g$ "(in the context of hypothesis $(a)$ of Theorem 2$)$ is replaced by " $\preccurlyeq$-compatibility of $f$ and $g$ ";

- "continuity of $f$ and $g$ " is replaced by the relatively weaker notion " $\preccurlyeq$-continuity of $f$ and $g^{\prime \prime}$;

- "continuity of $f$ " (in the context of hypothesis ( $a$ ) of Theorem 2) is replaced by the relatively weaker notion “ $\preccurlyeq$-continuity of $f$ ". Further, this notion is also not necessary as it can alternatively be replaced by " $(g, d)$-self-closedness of $\preccurlyeq$ ”;

- "regularity of $(X, d, M)$ " (in the context of hypothesis $(b)$ of Theorem 2) can be replaced by relatively weaker notion namely: " $(g, d)$-self-closedness of $\preccurlyeq$ ". Further, this notion is also not necessary as it can alternatively be replaced by " $(g, \preccurlyeq)$-continuity of $f$ " or "continuity of $f$ and $g^{\prime \prime}$;

- "closedness of whole subspace $g(X)$ " (in the context of hypothesis (b) of Theorem 2) is also replaced by " $\preccurlyeq$-closedness of any subset $Y$ with $f(X) \subseteq Y \subseteq g(X)$ ".

The following consequence of Theorem 5 and Corollary 1 is immediate.

Corollary 3 Theorem 5 (also Corollary 1 ) remains true if the local $f$-transitivity of $\mathcal{R}$ is replaced by one of the following conditions while retaining the rest of the hypotheses:

(i) $\mathcal{R}$ is transitive, 
(ii) $\mathcal{R}$ is $f$-transitive,

(iii) $\mathcal{R}$ is $g$-transitive,

(iv) $\mathcal{R}$ is locally transitive.

In view of Remarks 2-5, we conclude that Theorem 5 (also Corollaries 1, 2, and 3) remains true if the usual metrical terms of completeness, closedness, compatibility, continuity, and $g$-continuity are used instead of their respective $\mathcal{R}$-analogues.

Setting $g=I$, the identity mapping on $X$, in Theorem 5 , we obtain the corresponding fixed point result contained in [7].

Here we can point out that the proof of Theorem 4 can be carried out on the lines of Theorem 5 with $\varphi(t)=\alpha t(\alpha \in[0,1))$ even without using the following hypotheses (utilized in Theorem 5):

(i) $\mathcal{R}$ is locally $f$-transitive (in assumption (b)),

(ii) $\mathcal{R}$ is $(f, g)$-compatible (in assumptions $(e 3)$ and $\left(e^{\prime} 2\right)$ ).

Recall that condition (i) is utilized to prove the Cauchy property of $\left\{g x_{n}\right\}$ (in Theorem 5). In this case, proceeding on the lines of the proof of Theorem 5 , we have

$$
d\left(g x_{n+1}, g x_{n+2}\right)=d\left(f x_{n}, f x_{n+1}\right) \leq \alpha d\left(g x_{n}, g x_{n+1}\right) \quad \forall n \in \mathbb{N}_{0},
$$

so that

$$
d\left(g x_{n}, g x_{n+1}\right) \leq \alpha^{n} d\left(g x_{0}, g x_{1}\right) \quad \forall n \in \mathbb{N}_{0} .
$$

Now, using the standard techniques, we can show that $\left\{g x_{n}\right\}$ is a Cauchy sequence. Here there is no need to use condition (i), as contractivity condition is not necessary for $d\left(g x_{m_{k}}, g x_{n_{k}}\right)$.

In this case, $\varphi(0)=0$, and therefore by Corollary $2, \mathcal{R}$ is $(f, g)$-compatible. Hence condition (ii) is vacuously met out. Finally, we can accomplish the proof of Theorem 4 by proceeding on the lines of the proof of Theorem 5 (see the proof of Theorem 4.1 in [7]).

\section{Uniqueness results}

In this section, we present the results regarding the uniqueness of a point of coincidence, coincidence point, and a common fixed point corresponding to some earlier results. Recall that two self-mappings $f$ and $g$ defined on a nonempty set $X$ are called weakly compatible if $f(x)=g(x)$ implies $f(g x)=g(f x)$ for all $x \in X$.

Theorem 6 In addition to the hypotheses of Theorem 5, assume that the following conditions hold:

$$
\left(u_{1}\right): f(X) \text { is }\left.\mathcal{R}\right|_{g(X)} ^{s} \text { connected, and }
$$

$\left(u_{2}\right): \mathcal{R}$ is $(f, g)$-compatible.

Then $f$ and $g$ have a unique point of coincidence.

Proof In view of Theorem 5, let $\bar{x}$ and $\bar{y}$ be two points of coincidence of $f$ and $g$. Then there exist $x, y \in X$ such that

$$
\bar{x}=g(x)=f(x) \quad \text { and } \quad \bar{y}=g(y)=f(y) .
$$


We need to prove that $\bar{x}=\bar{y}$. Since $f(x), f(y) \in f(X) \subseteq g(X)$, by assumption $\left(u_{1}\right)$ there exists a path (say $\left\{g z_{0}, g z_{1}, g z_{2}, \ldots, g z_{k}\right\}$ ) of some finite length $k$ in $\left.\mathcal{R}\right|_{g(X)} ^{s}$ from $f(x)$ to $f(y)$ (where $\left.z_{0}, z_{1}, z_{2}, \ldots, z_{k} \in X\right)$. By (24), without loss of generality, we may set $z_{0}=x$ and $z_{k}=y$. Thus we obtain

$$
\left.\left[g z_{i}, g z_{i+1}\right] \in \mathcal{R}\right|_{g(X)} \quad \text { for each } i(0 \leq i \leq k-1)
$$

Define the constant sequences $z_{n}^{0}=x$ and $z_{n}^{k}=y$. Using (24), we have $g\left(z_{n+1}^{0}\right)=f\left(z_{n}^{0}\right)=\bar{x}$ and $g\left(z_{n+1}^{k}\right)=f\left(z_{n}^{k}\right)=\bar{y}$ for $n \in \mathbb{N}_{0}$. Put $z_{0}^{1}=z_{1}, z_{0}^{2}=z_{2}, \ldots, z_{0}^{k-1}=z_{k-1}$. As $f(X) \subseteq g(X)$, on the lines similar to proof of Theorem 5 , we can define sequences $\left\{z_{n}^{1}\right\},\left\{z_{n}^{2}\right\}, \ldots,\left\{z_{n}^{k-1}\right\}$ in $X$ such that $g\left(z_{n+1}^{1}\right)=f\left(z_{n}^{1}\right)$ and $g\left(z_{n+1}^{2}\right)=f\left(z_{n}^{2}\right), \ldots, g\left(z_{n+1}^{k-1}\right)=f\left(z_{n}^{k-1}\right)$ for $n \in \mathbb{N}_{0}$. Hence we obtain

$$
g\left(z_{n+1}^{i}\right)=f\left(z_{n}^{i}\right) \quad \forall n \in \mathbb{N}_{0} \text { and } i(0 \leq i \leq k) .
$$

Now we assert that

$$
\left[g z_{n}^{i}, g z_{n}^{i+1}\right] \in \mathcal{R} \quad \forall n \in \mathbb{N}_{0} \text { and } i(0 \leq i \leq k-1) .
$$

We prove this assertion by induction. It follows from (25) that (27) holds for $n=0$. Assume that (27) holds for $n=r>0$, that is,

$$
\left[g z_{r}^{i}, g z_{r}^{i+1}\right] \in \mathcal{R} \quad \text { for each } i(0 \leq i \leq k-1)
$$

Since $\mathcal{R}$ is $(f, g)$-closed, by Proposition 2, we obtain

$$
\left[f z_{r}^{i}, f z_{r}^{i+1}\right] \in \mathcal{R} \quad \text { for each } i(0 \leq i \leq k-1)
$$

which by (26) implies

$$
\left[g z_{r+1}^{i}, g z_{r+1}^{i+1}\right] \in \mathcal{R} \quad \text { for each } i(0 \leq i \leq k-1)
$$

It follows that (27) holds for $n=r+1$. Therefore, by induction, (27) holds for all $n \in \mathbb{N}_{0}$. Now, for all $n \in \mathbb{N}_{0}$ and $i(0 \leq i \leq k-1)$, define $t_{n}^{i}:=d\left(g z_{n}^{i}, g z_{n}^{i+1}\right)$. We claim that

$$
\lim _{n \rightarrow \infty} t_{n}^{i}=0 \quad \text { for each } i(0 \leq i \leq k-1)
$$

Fix $i$. Then two cases arise. Firstly, assume that $t_{n_{0}}^{i}:=d\left(g z_{n_{0}}^{i}, g z_{n_{0}}^{i+1}\right)=0$ for some $n_{0} \in \mathbb{N}_{0}$. Then by assumption $\left(u_{2}\right)$ we obtain $d\left(f z_{n_{0}}^{i}, f z_{n_{0}}^{i+1}\right)=0$. Consequently, using (26), we get $t_{n_{0+1}}^{i}=d\left(g z_{n_{0}+1}^{i}, g z_{n_{0}+1}^{i+1}\right)=d\left(f z_{n_{0}}^{i}, f z_{n_{0}}^{i+1}\right)=0$. Thus by induction we get $t_{n}^{i}=0$ for all $n \geq n_{0}$, yielding thereby $\lim _{n \rightarrow \infty} t_{n}^{i}=0$. On the other hand, assume that $t_{n}>0$ for all $n \in \mathbb{N}_{0}$. Then, using (26), (27), assumption (d), and Proposition 6, we obtain

$$
t_{n+1}^{i}=d\left(g z_{n+1}^{i}, g z_{n+1}^{i+1}\right)=d\left(f z_{n}^{i}, f z_{n}^{i+1}\right) \leq \varphi\left(d\left(g z_{n}^{i}, z_{n}^{i+1}\right)\right)=\varphi\left(t_{n}^{i}\right)
$$

so that

$$
t_{n+1}^{i} \leq \varphi\left(t_{n}^{i}\right) .
$$


Hence by Lemma 1 we obtain $\lim _{n \rightarrow \infty} t_{n}^{i}=0$. Therefore, in both the cases, (28) is proved for each $i(0 \leq i \leq k-1)$. Using the triangle inequality and (28), we obtain

$$
d(\bar{x}, \bar{y}) \leq t_{n}^{0}+t_{n}^{1}+\cdots+t_{n}^{k-1} \rightarrow 0 \quad \text { as } n \rightarrow \infty \quad \Longrightarrow \quad \bar{x}=\bar{y}
$$

Corollary 4 Theorem 6 remains true if we replace condition $\left(u_{1}\right)$ by one of the following conditions while retaining the rest of the hypotheses:

$\left.\left(u_{1}^{\prime}\right) \mathcal{R}\right|_{f(X)}$ is complete, and

$\left(u_{1}^{\prime \prime}\right) f(X)$ is $\left.\mathcal{R}\right|_{g(X)} ^{s}$-directed.

Theorem 7 In addition to the hypotheses of Theorem 6, suppose that the following condition holds:

$\left(u_{3}\right)$ : one off and $g$ is one-to-one.

Then $f$ and $g$ have a unique coincidence point.

Theorem 8 In addition to hypotheses ( $\left.e^{\prime}\right)$ of Theorem 6, suppose that the following condition holds:

$\left(u_{4}\right): f$ and $g$ are weakly compatible.

Then $f$ and $g$ have a unique common fixed point.

The proofs of Corollary 4 and of Theorems 7 and 8 are similar to those of Corollary 4.6 and of Theorems 4.7 and 4.8, respectively, which are contained in [7].

\section{An illustrative example}

In this section, we construct an example to highlight the worth and realized improvements in our newly proved results.

Example 1 Let $X=[0, \infty)$ with usual metric $d$ and binary relation $\mathcal{R}=\{(0,0),(0,1),(1,0)$, $(1,1),(3,0)\}$. Notice that, $\mathcal{R}$ is neither transitive nor $g$-transitive, but it is locally $f$ transitive. Define the pair of self-mappings $f$ and $g$ on $X$ by

$$
f(x)=\left\{\begin{array}{ll}
0, & x \in[0,1], \\
1, & x \in(1, \infty),
\end{array} \text { and } g(x)= \begin{cases}{[x],} & x \in[0,1] \\
3, & x \in(1, \infty)\end{cases}\right.
$$

Clearly, $\mathcal{R}$ is $(f, g)$-closed. Let $Y=\{0,1\}$. Then $Y$ is $\mathcal{R}$-complete, and $f(X)=\{0,1\} \subseteq Y \subseteq$ $g(X)=\{0,1,3\}$. Define the function $\varphi:[0, \infty) \rightarrow[0, \infty)$ by $\varphi(t)=\frac{1}{2} t$. Then $\varphi \in \Omega$.

Take any $\left.\mathcal{R}\right|_{Y}$-preserving sequence $\left\{x_{n}\right\}$ such that $x_{n} \stackrel{d}{\longrightarrow} x$. As $\left.\left(x_{n}, x_{n+1}\right) \in \mathcal{R}\right|_{Y}$ for all $n \in \mathbb{N}$, there exists $N \in \mathbb{N}$ such that $x_{n}=x \in\{0,1\}$ for all $n \geq N$. Therefore we can choose a subsequence $\left\{x_{n_{k}}\right\}$ of the sequence $\left\{x_{n}\right\}$ such that $x_{n_{k}}=x$ for all $k \in \mathbb{N}$, which amounts to saying that $\left.\left[x_{n_{k}}, x\right] \in \mathcal{R}\right|_{Y}$ for all $k \in \mathbb{N}$. Hence $\left.\mathcal{R}\right|_{Y}$ is $d$-self-closed. We can easily see that contraction condition $(d)$ and the remaining hypotheses of Theorem 5 are also satisfied. Consequently, in view of Theorem $5, f$ and $g$ have a coincidence point (namely, $x=0$ ).

Furthermore, hypotheses $\left(u_{1}\right),\left(u_{2}\right)$, and $\left(u_{4}\right)$ of Theorem 8 also hold. Thus all the hypotheses of Theorem 8 are satisfied, and hence $f$ and $g$ have a unique common fixed point (namely, $x=0$ ). 
Note that the present example cannot be covered by Karapinar et al. [1] and Erhan et al. [2] (i.e., Theorems 1 and 3, respectively), which substantiate the utility of Theorem 5 over Theorems 1 and 3.

\section{Conclusions}

In view of our newly proved results, we conclude that under relation-theoretic linear contraction, merely an arbitrary binary relation is required. If we extend such results to $\varphi$ contractions (under the family $\Omega$ ), then a weaker version of near-order is required, namely, a "locally $f$-transitive antisymmetric" (or, more appropriately, "locally $f$-transitive $(f . g)$ compatible)" binary relation. Particularly, in case $\varphi(0)=0$ or $g$ is one-to-one, a "locally $f$-transitive binary relation" is sufficient.

Karapinar et al. [1] observed that the notion of a transitive $F$-closed (or $F$-invariant) set is equivalent to the concept of a preordered set. To do this, given a nonempty subset $M^{n}$ of $X^{2 n}$, they defined the following relations:

- $n=2:(x, y) \sqsubseteq_{M^{2}}(u, v) \Longleftrightarrow\left[(x, y)=(u, v)\right.$ or $\left.(u, v, x, y) \in M^{2}\right]$;

- $n=3:(x, y, z) \sqsubseteq_{M^{3}}(u, v, w) \Longleftrightarrow\left[(x, y, z)=(u, v, w)\right.$ or $\left.(u, v, w, x, y, z) \in M^{3}\right]$.

For brevity, we consider $n=1$ and denote $M^{1}=M$. Then we analogously have

$$
x \sqsubseteq_{M} u \quad \Leftrightarrow \quad x=u \quad \text { or } \quad(u, x) \in M .
$$

Using this relation, they proved that (see Lemma 39 in [1])

(1) $\sqsubseteq_{M}$ is reflexive for whatever $M$,

(2) $M$ satisfies the transitive property if and only if $\bigsqcup_{M}$ is a preorder on $X$.

Actually, Karapinar et al. [1] defined $\beth_{M}$ (the dual relation of $\sqsubseteq_{M}$ ) as the reflexive closure of $M$, which enlarges $M$, not equivalent, that is,

$$
\begin{aligned}
& \sqsupseteq_{M}=M \cup \triangle_{X}=M^{\#}, \\
& \text { i.e., } \quad \sqsupseteq_{M}=\left(M^{\#}\right)^{-1} .
\end{aligned}
$$

Using this relation, they succeeded to prove such results in Lemma 39 of [1].

Therefore, if we redefine $x \sqsubseteq u \Longleftrightarrow(x, u) \in M$, then it is equivalent to say that $\sqsubseteq:=M$.

\section{Acknowledgements}

The last author is thankful to University Grant Commission, New Delhi, Government of India, for the financial support in the form of MANF (Moulana Azad National Fellowship). All the authors are thankful to three anonymous learned referees for their encouraging comments on the earlier version of the manuscript.

\section{Funding}

Not applicable.

Abbreviations

Not applicable.

Availability of data and materials

Not applicable.

Competing interests

The authors declare that they have no competing interests. 


\section{Publisher's Note}

Springer Nature remains neutral with regard to jurisdictional claims in published maps and institutional affiliations.

\section{Received: 1 November 2018 Accepted: 25 January 2019 Published online: 11 February 2019}

\section{References}

1. Karapinar, E., Roldán, A., Shahzad, N., Sintunavarat, W.: Discussion of coupled and tripled coincidence point theorems for $\varphi$-contractive mappings without the mixed g-monotone property. Fixed Point Theory Appl. 2014, 92 (2014)

2. Erhan, I.M., Roldán-López-de-Hierro, A.F., Shahzad, N.: Some remarks about the existence of coupled g-coincidence points. J. Inequal. Appl. 2015, 52 (2015)

3. Banach, S.: Sur les opérations dans les ensembles abstraits et leur application aux équations intégrales. Fundam Math. 3, 133-181 (1922)

4. Falset, J.G., Mleșnitşe, O.: Coincidence problems for generalized contractions. Appl. Anal. Discrete Math. 8(1), 1-15 (2014)

5. Petrușel, A., Petrușel, G., Xiao, Y.B., Yao, J.C.: Fixed point theorems for generalized contractions with applications to coupled fixed point theory. J. Nonlinear Convex Anal. 19(1), 71-88 (2018)

6. Agarwal, R.P., El-Gebeily, M.A., O'Regan, D.: Generalized contractions in partially ordered metric spaces. Appl. Anal. 87(1), 109-116 (2008)

7. Alam, A., Imdad, M.: Relation-theoretic metrical coincidence theorems. Filomat 31(14), 4421-4439 (2017)

8. Rashid, T., Alharbi, N., Khan, Q.H., Aydi, H., Özel, C.: Order-theoretic metrical coincidence theorems involving $(\phi, \psi)$-contractions. J. Math. Anal. 10(5), 119-135 (2018)

9. Petruşel, A., Petruşel, G., Yao, J.C.: Fixed point and coincidence point theorems in b-metric spaces with applications. Appl. Anal. Discrete Math. 11(1), 199-215 (2017)

10. Khan, M.S., Berzig, M., Chandok, S.: Fixed point theorems in bimetric space endowed with binary relation and applications. Miskolc Math. Notes 16(2), 939-951 (2015)

11. Roldán-López-de-Hierro, A.F., Shahzad, N.: Some fixed/coincidence point theorems under $(\psi, \varphi)$-contractivity conditions without an underlying metric structure. Fixed Point Theory Appl. 2014, 218 (2014)

12. Ran, A.C.M., Reurings, M.C.B.: A fixed point theorem in partially ordered sets and some applications to matrix equations. Proc. Am. Math. Soc. 132(5), 1435-1443 (2004)

13. Nieto, J.J., Rodríguez-López, R.: Contractive mapping theorems in partially ordered sets and applications to ordinary differential equations. Order 22(3), 223-239 (2005)

14. Harjani, J., Sadarangani, K.: Fixed point theorems for weakly contractive mappings in partially ordered sets. Nonlinear Anal. 71(7-8), 3403-3410 (2009)

15. Harandi, A.A., Emami, H.: A fixed point theorem for contraction type maps in partially ordered metric spaces and application to ordinary differential equations. Nonlinear Anal. 72(5), 2238-2242 (2010)

16. Altun, I., Rakočević, V.: Ordered cone metric spaces and fixed point results. Comput. Math. Appl. 60, 1145-1151 (2010)

17. Jachymski, J.: Equivalent conditions for generalized contractions on (ordered) metric spaces. Nonlinear Anal. 74(3), 768-774 (2011)

18. Alam, A., Khan, A.R., Imdad, M.: Some coincidence theorems for generalized nonlinear contractions in ordered metric spaces with applications. Fixed Point Theory Appl. 2014, 216 (2014)

19. Shoaib, A., Arshad, M., Beg, l.: Fixed points of contractive dominated mappings in an ordered quasi-partial metric spaces. Matematiche LXX, 283-294 (2015)

20. Turinici, M.: Linear contractions in product ordered metric spaces. Ann. Univ. Ferrara 59, 187-198 (2013)

21. Ben-El-Mechaiekh, H.: The Ran-Reurings fixed point theorem without partial order: a simple proof. J. Fixed Point Theory Appl. 16, 373-383 (2014)

22. Turinici, M.: Ran-Reurings fixed point results in ordered metric spaces. Libertas Math. 31, 49-55 (2011)

23. Turinici, M.: Nieto-Lopez theorems in ordered metric spaces. Math. Stud. 81(1-4), 219-229 (2012)

24. Ghods, S., Gordji, M.E., Ghods, M., Hadian, M.: Comment on "Coupled fixed point theorems for nonlinear contractions in partially ordered metric spaces" [Lakshmikantham and Ćirić, Nonlinear Anal. TMA 70 (2009) 4341-4349]. J. Comput. Anal. Appl. 14(5), 958-966 (2012)

25. Samet, B., Turinici, M.: Fixed point theorems on a metric space endowed with an arbitrary binary relation and applications. Commun. Math. Anal. 13(2), 82-97 (2012)

26. Alam, A., Imdad, M.: Relation-theoretic contraction principle. J. Fixed Point Theory Appl. 17(4), 693-702 (2015)

27. Ayari, M..., Berzig, M., Kédim, l.: Coincidence and common fixed point results for $\beta$-quasi contractive mappings on metric spaces endowed with binary relation. Math. Sci. 10(3) (2016)

28. Roldán-López-de-Hierro, A.F.: A unified version of Ran and Reuring's theorem and Nieto and R. Rodríguez-López's theorem and low-dimensional generalizations. Appl. Math. Inf. Sci. 10(2), 383-393 (2016)

29. Roldán-López-de-Hierro, A.F., Shahzad, N.: Fixed point theorems by combining Jleli and Samet's, and Branciari's inequalities. J. Nonlinear Sci. Appl. 9, 3822-3849 (2016)

30. Shahzad, N., Roldán-López-de-Hierro, A.F., Khojasteh, F.: Some new fixed point theorems under $(\mathcal{A}, \mathcal{S})$-contractivity conditions. Rev. R. Acad. Cienc. Exactas Fís. Nat., Ser. A Mat. 111, 307-324 (2017)

31. Shahzad, N., Karapinar, E., Roldán-López-de-Hierro, A.F:: On some fixed point theorems under $(\alpha, \psi, \varphi)$-contractivity conditions in metric spaces endowed with transitive binary relations. Fixed Point Theory Appl. 2015, 124 (2015)

32. Berzig, M., Karapinar, E.: Fixed point results for $(\alpha \psi, \beta \varphi)$-contractive mappings for a generalized altering distance. Fixed Point Theory Appl. 2013, 205 (2013)

33. Berzig, M., Karapinar, E., Roldán-López-de-Hierro, A.F.: Discussion on generalized- $(\alpha \psi, \beta \varphi)$-contractive mappings via generalized altering distance function and related fixed point theorems. Abstr. Appl. Anal. 2014, s259768 (2014)

34. Turinici, M.: Contractive maps in locally transitive relational metric spaces. Sci. World J. 2014, 169358 (2014)

35. Turinici, M.: Contractive operators in relational metric spaces. In: Handbook of Functional Equations. Springer Optimization and Its Applications, vol. 95, pp. 419-458. Springer, Berlin (2014)

36. Alam, A., Arif, M., Imdad, M.: Metrical fixed point theorems via locally finitely T-transitive binary relations under certain control functions. Miskolc Math. Notes (accepted) 
37. Alam, A., Imdad, M.: Nonlinear contractions in metric spaces under locally T-transitive binary relations. Fixed Point Theory 19(1), 13-24 (2018)

38. Samet, B., Vetro, C.: Coupled fixed point, F-invariant set and fixed point of N-order. Ann. Funct. Anal. 1(2), 46-56 (2010)

39. Kutbi, M.A., Roldán, A., Sintunavarat, W., Martinez-Moreno, J., Roldan, C.: F-Closed sets and coupled fixed point theorems without the mixed monotone property. Fixed Point Theory Appl. 2013, 330 (2013)

40. Lakshmikantham, V., Ćirić, L.: Coupled fixed point theorems for nonlinear contractions in partially ordered metric spaces. Nonlinear Anal. 70, 4341-4349 (2009)

41. Lipschutz, S.: Schaum's Outlines of Theory and Problems of Set Theory and Related Topics. McGraw-Hill, New York (1964)

42. Maddux, R.D.: Relation Algebras. Studies in Logic and the Foundations of Mathematics, vol. 150. Elsevier, Amsterdam (2006)

43. Flaška, V., Ježek, J., Kepka, T., Kortelainen, J.: Transitive closures of binary relations I. Acta Univ. Carol., Math. Phys. 48(1), 55-69 (2007)

44. Skala, H.L.: Trellis theory. Algebra Univers. 1, 218-233 (1971)

45. Stouti, A., Maaden, A.: Fixed points and common fixed points theorems in pseudo-ordered sets. Proyecciones 32(4), 409-418 (2013)

46. Kolman, B., Busby, R.C., Ross, S.: Discrete Mathematical Structures, 3rd edn. PHI Pvt. Ltd., New Delhi (2000)

47. Roldán-López-de-Hierro, A.F., Karapinar, E., de la Sen, M.: Coincidence point theorems in quasi-metric spaces without assuming the mixed monotone property and consequences in G-metric spaces. Fixed Point Theory Appl. 2014, 184 (2014)

48. Boyd, D.W., Wong, J.S.W.: On nonlinear contractions. Proc. Am. Math. Soc. 20, 458-464 (1969)

49. Jotic, N.: Some fixed point theorems in metric spaces. Indian J. Pure Appl. Math. 26, 947-952 (1995)

50. Haghi, R.H., Rezapour, Sh., Shahzad, N.: Some fixed point generalizations are not real generalizations. Nonlinear Anal. 74, 1799-1803 (2011)

\section{Submit your manuscript to a SpringerOpen ${ }^{\circ}$ journal and benefit from:}

- Convenient online submission

Rigorous peer review

Open access: articles freely available online

- High visibility within the field

- Retaining the copyright to your article

Submit your next manuscript at $\gg$ springeropen.com 